Homology, Homotopy and Applications, vol.17(2), 2015, pp.13-24

\title{
ON HOMOTOPY CATEGORIES OF GORENSTEIN MODULES: COMPACT GENERATION AND DIMENSIONS
}

\author{
NAN GAO \\ (communicated by Claude Cibils)
}

\begin{abstract}
Let $A$ be a virtually Gorenstein algebra of finite CM-type. We establish a duality between the subcategory of compact objects in the homotopy category of Gorenstein projective left $A$-modules and the bounded Gorenstein derived category of finitely generated right $A$-modules. Let $R$ be a two-sided noetherian ring such that the subcategory of Gorenstein flat modules $R-\mathcal{G F}$ is closed under direct products. We show that the inclusion $K(R-\mathcal{G F}) \rightarrow K(R$-Mod) of homotopy categories admits a right adjoint. We introduce the notion of Gorenstein representation dimension for an algebra of finite CM-type, and give a lower bound by the dimension of its bounded Gorenstein derived category.
\end{abstract}

\section{Introduction}

Gorenstein projective modules and algebras of finite CM-type are of interest in the representation theory of algebras, Gorenstein homological algebra, and in the theory of singularity categories (see, e.g., [AM, EJ, CFH, Buc, Be]).

Triangulated categories, especially, derived categories, introduced by Grothendieck and Verdier [Ver], have been playing an increasingly important role in various areas of mathematics, including representation theory, algebraic geometry, and mathematical physics. In the last decade, some of the progress has been made on Brown representability in homotopy theory (see, e.g., [CN, BS2]). There are several theorems telling us about the existence and uniqueness of model structures for large classes of triangulated categories ([LO, S]). To be able to use the Brown representability theorem, a triangulated category needs a basic property of compact generation [N2]. Later, Krause [K1] and Jørgensen [J] established relations between the compact generation of a triangulated category and the existence of dualizing complexes, respectively.

A major topic of current interest is the compact generation of the homotopy category of projective modules, $K(R-\mathcal{P})$, of a ring $R$. Jørgensen $[\mathrm{J}]$ has shown that for any reasonably nice ring, $K(R-\mathcal{P})$ is compactly generated, and he also has established

Supported by the National Natural Science Foundation of China (Grant No. 11101259).

Received February 11, 2014, revised August 29, 2014, January 25, 2015; published on July 13, 2015. 2010 Mathematics Subject Classification: 18G25.

Key words and phrases: Gorenstein projective module, Gorenstein flat module, compactly generated homotopy category, Gorenstein representation dimension.

Article available at http://dx.doi.org/10.4310/HHA.2015.v17.n2.a2

Copyright (C) 2015, International Press. Permission to copy for private use granted. 
a duality between its subcategory of compact objects and the bounded derived category of finitely presented right modules. This was generalized by Neeman in [N4] to arbitrary rings. He has proved that $K(R-\mathcal{P})$ is always $\aleph_{1}$-compactly generated for any ring $R$.

These results raise questions on the homotopy category of Gorenstein projective modules:

(1) When is the homotopy category of Gorenstein projective modules compactly generated? What is the subcategory of compact objects? How is it related to the Gorenstein derived category of the corresponding ring introduced by Gao and Zhang [GZ2]?

(2) In this case, what is the relation between the subcategory of compact objects in the homotopy category of Gorenstein projective modules and the subcategory of compact objects in the homotopy category of projective modules?

We completely answer both these questions for virtually Gorenstein algebras of finite CM-type (Theorems 3.2 and 3.6).

It is known that projective modules and flat modules are the same over artin algebras. However, it is not true in general. For an arbitrary ring $R$, Neeman [N5] has shown the inclusion of the homotopy category of flat $R$-modules, $K(R-\mathcal{F})$, into $K(R$-Mod $)$ has a right adjoint. In [K2] a criterion has been given for the existence of right approximations in cocomplete additive categories, which is used to construct adjoint functors between homotopy categories.

These results raise questions on the homotopy category of Gorenstein flat modules:

(3) Can we establish a pair of adjoint functors between the homotopy category of Gorenstein flat modules and the homotopy category of a ring?

We completely answer question (3) for two-sided noetherian rings such that the subcategories of Gorenstein flat modules are closed under direct products (Theorem 3.8).

The concept of dimension of a triangulated category has been introduced by Rouquier [Ro]. He defined and studied the dimension for a triangulated category in order to prove that the representation dimension of an algebra can be arbitrarily large. He has also shown that for an algebra the relations among the global dimension, Auslander's representation dimension, and the dimension of the bounded derived category. See [KK, O, BIKO] for more information on these topics.

These results raise questions on the bounded Gorenstein derived category and the homotopy category of Gorenstein projective modules:

(4) What is the analogue of Auslander's representation dimension in Gorenstein homological algebra? Can we establish the relations between it and the dimension of its bounded Gorenstein derived category?

We provide such analogues and relate them by a chain of inequalities (Definition 4.2 and Theorem 4.6).

Let us end this introduction by mentioning that model structures for Gorenstein derived categories and homotopy categories of Gorenstein projective modules are investigated, which will appear in a coming paper. 


\section{Preliminaries}

In this section, we fix notation and recall the main concepts to be used.

Let $A$ be an artin algebra. Denote by $A$-Mod (resp. $A$-mod) the category of (resp. finitely generated) left $A$-modules, and by $A$-P (resp. $A$-proj) the full subcategory of (resp. finitely generated) projective $A$-modules. An $A$-module $M$ is said to be Gorenstein projective in $A$-Mod (resp. $A$-mod) if there is an exact sequence $P^{\bullet}=\cdots \longrightarrow$ $P^{-1} \longrightarrow P^{0} \stackrel{d^{0}}{\longrightarrow} P^{1} \longrightarrow P^{2} \longrightarrow \cdots$ in $A$-P (resp. $A$-proj) with $\operatorname{Hom}_{A}\left(P^{\bullet}, Q\right)$ exact for any $A$-module $Q$ in $A$-P (resp. $A$-proj), such that $M \cong \operatorname{ker} d^{0}$ (see [EJ, Definition 10.2.1]). Denote by $A-\mathcal{G P}$ (resp. $A-\mathcal{G}$ proj) the full subcategory of Gorenstein projective modules in $A$-Mod (resp. $A$-mod). For the notion of a Gorenstein injective module we refer to [EJ, Definition 10.1.1]. We denote the subcategory of Gorenstein injective $A$-modules by $A-\mathcal{G I}$.

Now recall the notion of a Gorenstein flat module. Denote by $A-\mathcal{F}$ the full subcategory of flat $A$-modules. An $A$-module $M$ is said to be Gorenstein flat in $A$-Mod, if there is an exact sequence $F^{\bullet}=\cdots \longrightarrow F^{-1} \longrightarrow F^{0} \stackrel{d^{0}}{\longrightarrow} F^{1} \longrightarrow F^{2} \longrightarrow \cdots$ in $A-\mathcal{F}$ with $I \otimes_{A} F^{\bullet}$ exact for any injective right $A$-module $I$, such that $M \cong \operatorname{ker} d^{0}$ (see [EJ, Definition 10.3.1]). Denote by $A-\mathcal{G F}$ the full subcategory of Gorenstein flat modules in $A$-Mod.

A proper $A$ - $\mathcal{G}$ proj-resolution of an $A$-module $M$ in $A$-mod is an exact sequence $E^{\bullet}=\cdots \longrightarrow G_{1} \longrightarrow G_{0} \longrightarrow M \longrightarrow 0$ such that all $G_{i} \in A-\mathcal{G}$ proj, and that $\operatorname{Hom}_{A}(G$, $\left.E^{\bullet}\right)$ stays exact for each $G \in A-\mathcal{G}$ proj. The second requirement guarantees the uniqueness of such a resolution in the homotopy category (the Comparison Theorem; see [EJ, p.169]). The coproper $A-\mathcal{G}$ proj-resolution is defined dually.

The Gorenstein projective dimension $\mathcal{G} p \operatorname{dim} M$ of $M$ in $A$-mod is defined to be the smallest integer $n \geqslant 0$ such that there is an exact sequence $0 \longrightarrow G_{n} \longrightarrow \cdots \longrightarrow$ $G_{1} \longrightarrow G_{0} \longrightarrow M \longrightarrow 0$ with all $G_{i} \in A-\mathcal{G}$ proj, if it exists, and $\mathcal{G} p \operatorname{dim} M=\infty$ if there is no such exact sequence of finite length.

A complex $C^{\bullet}$ of (finitely generated) $A$-modules is $A-\mathcal{G P}$ - (resp. $A-\mathcal{G}$ proj-) acyclic, if the complex $\operatorname{Hom}_{A}\left(G, C^{\bullet}\right)$ is acyclic for any $G \in A-\mathcal{G P}$ (resp. $A-\mathcal{G}$ proj). It is also called proper exact, for example, in $[\mathrm{AM}]$. A chain map $f^{\bullet}: X^{\bullet} \longrightarrow Y^{\bullet}$ is an $A$-G projquasi-isomorphism if $\operatorname{Hom}_{A}\left(G, f^{\bullet}\right)$ is a quasi-isomorphism for any $G \in A$-G proj; i.e., there are isomorphisms of abelian groups $\mathrm{H}^{n} \operatorname{Hom}_{A}\left(G, f^{\bullet}\right): \mathrm{H}^{n} \operatorname{Hom}_{A}\left(G, X^{\bullet}\right) \cong$ $\mathrm{H}^{n} \operatorname{Hom}_{A}\left(G, Y^{\bullet}\right), \forall n \in \mathbb{Z}, \forall G \in A$ - $\mathcal{G}$ proj.

In the following, we denote by $C(A$-Mod $)$ the category of complexes of $A$-modules in $A$-Mod, and by $C_{\text {gpac }}(A$-Mod $)$ the full subcategory of $C(A$-Mod $)$ consisting of $A-\mathcal{G} P$-acyclic complexes.

Following [GZ2, 2.3], the (bounded) Gorenstein derived category $D_{g p}(A$-Mod) (resp. $\left.D_{g p}^{b}(A)\right)$ of $A$ is defined as the Verdier quotient of the (bounded) homotopy category $K\left(A\right.$-Mod) (resp. $K^{b}(A$-mod) ) with respect to the triangulated subcategory $K_{\text {gpac }}\left(A\right.$-Mod) (resp. $K_{\text {gpac }}^{b}(A$-mod) $)$ of $A-\mathcal{G} P$ - (resp. $A$-G $\mathcal{G}$ proj-) acyclic complexes.

Recall from $[\mathrm{BH}, \mathrm{Be}]$ that an artin algebra $A$ is of finite CM-type if there are only finitely many isomorphism classes of finitely generated indecomposable Gorenstein projective $A$-modules. Suppose $A$ is an artin algebra of finite CM-type, that $G_{1}, \ldots, G_{n}$ are all the pairwise non-isomorphic indecomposable finitely generated 
Gorenstein projective $A$-modules, and $G=\bigoplus_{1 \leqslant i \leqslant n} G_{i}$. Set $\mathcal{G} p(A):=\operatorname{End}_{A}(G)^{\mathrm{op}}$, which we call the relative Auslander algebra of $A$. It is clear that $G$ is an $A-\mathcal{G} p(A)$-bimodule and $\mathcal{G} p(A)$ is an artin algebra ([ARS, p.27]). Denote by $\mathcal{G} p(A)$-mod the category of finitely generated left $\mathcal{G} p(A)$-modules. Recall from $[\mathrm{BR}]$ that an artin algebra $A$ is called virtually Gorenstein if $A-\mathcal{G} \mathcal{P}^{\perp}={ }^{\perp} A-\mathcal{G I}$, where $A-\mathcal{G} \mathcal{P}^{\perp}:=\left\{X \in A\right.$-Mod $\mid \operatorname{Ext}_{A}^{i}$ $(G, X)=0, \forall i>0$ and $\forall G \in A-\mathcal{G P}\}$ and ${ }^{\perp} A-\mathcal{G I}:=\left\{Y \in A\right.$-Mod $\mid \operatorname{Ext}_{A}^{i}(Y, I)=0$, $\forall i>0$ and $\forall I \in A-\mathcal{G I}\}$.

Let $\mathcal{T}$ be a triangulated category with arbitrary small coproducts. Recall from [N3, Definition 4.2.7] that an object $T \in \mathcal{T}$ is compact if the functor $\operatorname{Hom}_{\mathcal{T}}(T,-)$ preserves coproducts. The full subcategory of all compact objects in $\mathcal{T}$ will be denoted $\mathcal{T}^{c}$.

\section{The subcategory $K(A-\mathcal{G P})^{c}$ of compact objects}

In this section, we show that if $A$ is a virtually Gorenstein artin algebra of finite CM-type, then the subcategory of compact objects, $K(A-\mathcal{G P})^{c}$, of the homotopy category $K(A-\mathcal{G P})$ is triangular equivalent to the opposite category of the bounded Gorenstein derived category $D_{g p}^{b}\left(A^{\text {op }}\right)$ of $A^{o p}$. We also prove that if $R$ is a two-sided noetherian ring such that the category of Gorenstein flat $R$-modules, $R-\mathcal{G F}$, is closed under direct products, then the inclusion of the homotopy category of Gorenstein flat $R$-modules, $K(R-\mathcal{G} \mathcal{F})$, into the homotopy category $K(R$-Mod) admits a right adjoint.

Let $A$ be a virtually Gorenstein artin algebra of finite CM-type. Then $A^{\text {op }}$ is also a virtually Gorenstein artin algebra of finite CM-type. By [Be, Theorem 4.10 and Proposition 4.18] we know that $A-\mathcal{G P}=\operatorname{Add}(A-\mathcal{G}$ proj $)$ and $A^{\mathrm{op}}-\mathcal{G}$ proj is contravariantly finite in $A^{\mathrm{op}}$-mod. Denote by ()$^{*}$ the functor $\operatorname{Hom}_{A}(-, A)$.

Recall from $[\mathrm{GZ2}, 3.3]$ that $K^{-, g p b}\left(A^{\mathrm{op}}-\mathcal{G}\right.$ proj $)$ is defined be the full subcategory of the upper bounded homotopy category $K^{-}\left(A^{\mathrm{op}_{-}} \mathcal{G}_{\mathrm{proj}}\right)$ of $A^{\mathrm{op}}-\mathcal{G}_{\mathrm{proj}}$ by

$$
\begin{aligned}
K^{-, g p b}\left(A^{\mathrm{op}}-\mathcal{G} \text { proj }\right):=\left\{X^{\bullet} \in\right. & K^{-}\left(A^{\mathrm{op}_{-}} \mathcal{G}_{\text {proj }}\right) \mid \exists n=n\left(X^{\bullet}\right) \in \mathbb{Z}, \quad \text { such that } \\
& \left.H^{i} \operatorname{Hom}_{A^{\mathrm{op}}}\left(G, X^{\bullet}\right)=0, \quad \forall i \leqslant n, \quad \forall G \in A^{\mathrm{op}_{-}} \mathcal{G}_{\text {proj }}\right\} .
\end{aligned}
$$

Construction 3.1. Let $M$ be a finitely generated left $A$-module. Then there is a proper $A^{\text {op }} \mathcal{G}_{-}$proj-resolution $G^{\bullet}$ with $G^{\bullet}$ in $K^{-, g p b}\left(A^{\text {op }} \mathcal{G}_{-}\right.$proj $)$for $M^{*}$ because $A^{\text {op }} \mathcal{G}_{-}$proj is contravariantly finite in $A^{\mathrm{op}}$-mod, as stated above. It follows from [HJ2, Theorem 1.6] that $G^{\bullet *}$ is a coproper $A-\mathcal{G}$ proj-resolution of $M$. Set

$$
\mathcal{G}_{2}:=\left\{G^{\bullet *}[n] \mid M \in A \text {-mod, } n \in \mathbb{Z}\right\} .
$$

Theorem 3.2. $K(A-\mathcal{G P})$ is a compactly generated triangulated category with $\mathcal{G}_{2}$ as a set of compact generators. Moreover, there is a triangle-equivalence

$$
K(A-\mathcal{G P})^{c} \cong D_{g p}^{b}\left(A^{\mathrm{op}}\right)^{\mathrm{op}} .
$$

Proof. By [HJ1, Theorem 3.1], we get that $\mathcal{G}_{2}$ is a set of compact objects in $K(A-\mathcal{G P})$. Also, by the proof of [G2, Theorem 2.2] we get that $\mathcal{G}_{2}$ generates $K(A-\mathcal{G P})$. This implies that $K(A-\mathcal{G P})^{c}$ is a full subcategory of $K(A-\mathcal{G P})$ consisting of objects that are finitely built from objects $G^{\bullet}$ in $\mathcal{G}_{2}$, using shifts, distinguished triangles, and direct summands. 
Now we set $\mathcal{G}_{2}^{*}:=\left\{G^{\bullet *} \mid G^{\bullet} \in \mathcal{G}_{2}\right\}$. Denote by $\mathcal{D}$ the full subcategory of $K\left(A^{\text {op }}-\mathcal{G P}\right)$ consisting of objects that are finitely built from objects in $\mathcal{G}_{2}^{*}$. Since the canonical chain maps $G^{\bullet} \rightarrow G^{\bullet * *}$ and $G^{\bullet *} \rightarrow G^{\bullet * * *}$ are isomorphisms, it follows that

$$
K(A-\mathcal{G P})^{c} \rightleftarrows \mathcal{D}^{\text {op }}
$$

are quasi-inverse equivalences of triangulated categories.

Now we claim that $\mathcal{D}$ consists of the objects that are finitely built from proper $A^{\mathrm{op}}-\mathcal{G}$ proj-resolutions of all finitely generated $A^{\mathrm{op}}$-modules. Then by [GZ2, Theorem 3.6], we get that $\mathcal{D}$ is triangular equivalent to $D_{g p}^{b}\left(A^{\mathrm{op}}\right)$. This completes the proof.

Suppose that $N$ is a finitely generated $A^{\mathrm{op}}$-module, and let

$$
E^{\bullet}=\cdots \rightarrow E^{-2} \rightarrow E^{-1} \rightarrow E^{0} \rightarrow 0
$$

be a proper $A^{\mathrm{op}}-\mathcal{G}$ proj-resolution of $N$. Now

$$
\widetilde{E^{\bullet}}=\cdots \rightarrow E^{-3} \rightarrow E^{-2} \rightarrow 0 \rightarrow \cdots
$$

is a proper $A^{\text {op }} \mathcal{G}$ proj-resolution of $Z^{-1}\left(\widetilde{E^{\bullet}}\right)$, the $(-1)$ st cycle module of $E^{\bullet}$. Complete $E^{0 *} \rightarrow E^{-1 *}$ with its cokernel,

$$
E^{0 *} \rightarrow E^{-1 *} \rightarrow M \rightarrow 0 .
$$

Then $Z^{-1}\left(\widetilde{E^{\bullet}}\right)=M^{*}$. This implies that $\widetilde{E^{\bullet}}$ is in $\mathcal{G}_{2}^{*}$, and so $E^{\bullet}$ is in $\mathcal{G}_{2}^{*}$.

Corollary 3.3. Let $A$ be a virtually Gorenstein algebra of finite CM-type.

(1) There exists the recollement

$$
K(A-\mathcal{G P}) \underset{r}{\stackrel{\frac{l}{\zeta}}{\rightleftarrows}} K(A \text {-Mod }) \underset{\leftrightarrows}{\leftrightarrows}(A-\mathrm{Mod}) / K(A-\mathcal{G P}) .
$$

In particular, if $A$ is also Gorenstein, then there is the recollement of the form

$$
K(A-\mathcal{G P}) \underset{r}{\stackrel{i}{\rightleftarrows}} K(A \text {-Mod }) \underset{r}{\longleftarrow} K_{\text {gpac }}(A \text {-Mod }) .
$$

(2) There exists the right recollement

$$
K_{\text {gpac }}(A \text {-Mod }) \underset{r}{\stackrel{i}{\rightleftarrows}} K(A \text {-Mod }) \rightleftarrows D_{g p}(A \text {-Mod }) .
$$

and $D_{g p}(A$-Mod) has small Hom-sets. If $A$ is also Gorenstein, then there is the recollement of the form

$$
K_{\text {gpac }}(A \text {-Mod }) \underset{r}{\stackrel{i}{\rightleftarrows}} K(A \text {-Mod }) \underset{\leftrightarrows}{\longleftarrow} K(A-\mathcal{G P}) .
$$

Proof. (1) By Theorem 3.2, we get that $K(A-\mathcal{G P})$ is compactly generated. Since the inclusion $i$ naturally preserves coproducts and products, it follows from [N2, Theorem 4.1] that $i$ admits a right adjoint $r$, also a left adjoint $l$. So we get from 
[CPS1, Theorem 2.1] that there is the following recollement:

$$
K(A-\mathcal{G P}) \underset{r}{\stackrel{i}{\rightleftarrows}} K(A \text {-Mod }) \underset{\leftrightarrows}{\leftrightarrows} K(A \text {-Mod }) / K(A-\mathcal{G P})
$$

If $A$ is also Gorenstein, then by [G1, Theorem 2.7] there is a triangle equivalence

$$
K(A-\operatorname{Mod}) / K(A-\mathcal{G P}) \cong K_{\text {gpac }}(A-\mathrm{Mod}) .
$$

This completes the proof of (1).

(2) By [Be, Theorem 4.10], we get that every module in $A-\mathcal{G P}$ is a filtered colimit of modules in $A-\mathcal{G}$ proj. From this, it follows that $C_{\text {gpac }}(A$-Mod $)$ is closed under filtered colimits. By minor modifications of the proof of [K2, Lemma 2(4)], we get that $C_{\text {gpac }}(A$-Mod $)$ is closed under $\alpha$-pure subobjects for some regular cardinal $\alpha$. Thus by [K2, Theorem 4], we get that every complex in $K(A$-Mod) admits a right $K_{\text {gpac }}(A$-Mod)-approximation. Applying [N5, Proposition 1.4], it follows that the inclusion $i: K_{\text {gpac }}(A$ - Mod $) \rightarrow K(A$-Mod $)$ admits a right adjoint $r$. Therefore, we obtain from [Mi, Theorem 2.2] the following right recollement

$$
K_{\text {gpac }}(A \text {-Mod }) \underset{r}{\stackrel{i}{\rightleftarrows}} K(A \text {-Mod }) \rightleftarrows D_{\text {gp }}(A \text {-Mod })
$$

By [GZ1, Proposition I.1.3], we know that the right adjoint of the quotient functor $K(A$ - Mod $) \rightarrow D_{g p}(A$-Mod $)$ is fully faithful. Therefore, $D_{g p}(A$-Mod $)$ has small Homsets.

Since $A$ is virtually Gorenstein of finite CM-type, it follows from the proof of (1) that the inclusion $K(A-\mathcal{G P}) \rightarrow K(A$-Mod $)$ admits a right adjoint. Moreover, if $A$ is Gorenstein, then we get from $\left[\mathrm{G} 1\right.$, Theorem 2.7] that $K(A-\mathcal{G P})^{\perp}=K_{\text {gpac }}(A$-Mod), where $K(A-\mathcal{G P})^{\perp}=\left\{X^{\bullet} \in K(A\right.$-Mod $) \mid \operatorname{Hom}_{K(A \text {-Mod })}\left(G^{\bullet}, X^{\bullet}\right)=0$, for all $\left.G^{\bullet} \in K(A-\mathcal{G P})\right\}$. This means that the inclusion $K_{\text {gpac }}(A$-Mod $) \rightarrow K(A$-Mod $)$ admits a left adjoint and the composition $K(A$ - $\mathcal{G P}) \rightarrow K(A$-Mod $) \rightarrow D_{g p}(A$-Mod $)$ is a triangle-equivalence. This completes the proof of $(2)$.

Corollary 3.4. Let $A$ be a Gorenstein algebra of finite CM-type. Then the canonical functor $D_{g p}(A$-Mod $) \rightarrow D(A$-Mod $)$ admits left and right adjoints that are fully faithful. The left adjoint preserves compactness, and its restriction to $K^{b}(A$-proj) identifies with the inclusion $K^{b}(A$-proj $) \rightarrow K^{b}(A-\mathcal{G}$ proj $)$.

Proof. Since $A$ is Gorenstein of finite CM-type, it follows from Theorem 3.2 that $K(A-\mathcal{G P})$ is compactly generated and $K(A-\mathcal{G} P)^{c} \cong K^{b}(A-\mathcal{G}$ proj $)$. By Corollary 3.3(2), there is a triangle equivalence $D_{g p}(A-\mathrm{Mod}) \cong K(A-\mathcal{G} P)$. This implies that there is a triangle equivalence $D_{g p}(A \text {-Mod })^{c} \cong K^{b}(A-\mathcal{G}$ proj $)$. Note that the canonical functor $F: D_{g p}(A$-Mod $) \rightarrow D(A$-Mod $)$ preserves set-indexed coproducts and products. So we get from [N2, Theorem 4.1] that $F$ admits a left adjoint and a right adjoint, and also that the left adjoint preserves compactness. It follows from [N1, Theorem 2.1] that the restriction of this left adjoint to $K^{b}(A$-proj) identifies with the inclusion $K^{b}(A$-proj $) \rightarrow K^{b}(A-\mathcal{G}$ proj $)$. Since $F$ is a Verdier quotient functor, it follows from [GZ1, Proposition I.1.3] that these adjoints are fully faithful.

Next, we will compare the subcategory of compact objects in the homotopy category of projective modules with the subcategory of compact objects in the homotopy category of Gorenstein projective modules. We first recall the construction of compact 
generators of the homotopy category of projective $A$-modules for an artin algebra $A$ we will use.

Let $A$ be an artin algebra, and let $M$ be a finitely generated $A$-module. Then for $M^{*}$ there is a canonical quasi-isomorphism $P^{\bullet} \rightarrow M^{*}$ with $P^{\bullet}$ in the homotopy category $K^{-, b}\left(A^{\text {op }}\right.$-proj). We consider the collection of the form $P^{\bullet *}[n](n \in \mathbb{Z})$. Denote by $\mathcal{G}_{1}$ the set of one object from each such isomorphism class.

Lemma 3.5 ([J, Theorem 2.4 and 3.2]). The homotopy category $K(A-\mathcal{P})$ is a compactly generated triangulated category with $\mathcal{G}_{1}$ as a set of compact generators. Moreover, there is a triangle equivalence

$$
K(A-\mathcal{P})^{c} \cong D^{b}\left(A^{\mathrm{op}}\right)^{\mathrm{op}} .
$$

Theorem 3.6. Let $A$ be a virtually Gorenstein algebra of finite CM-type. Then

(1) $K(A-\mathcal{G P}) / K(A-\mathcal{P})$ is a compactly generated triangulated category;

(2) the localisation sequence of triangulated categories

$$
K(A-\mathcal{P}) \stackrel{i}{\hookrightarrow} K(A-\mathcal{G P}) \stackrel{q}{\rightarrow} K(A-\mathcal{G P}) / K(A-\mathcal{P})
$$

yields, by restriction to compact objects, a sequence of functors

$$
D^{b}\left(A^{\mathrm{op}}\right)^{\mathrm{op}} \rightarrow D_{g p}^{b}\left(A^{\mathrm{op}}\right)^{\mathrm{op}} \rightarrow(K(A-\mathcal{G P}) / K(A-\mathcal{P}))^{c} .
$$

Moreover, the induced functor

$$
F: D_{g p}^{b}\left(A^{\mathrm{op}}\right)^{\mathrm{op}} / D^{b}\left(A^{\mathrm{op}}\right)^{\mathrm{op}} \rightarrow(K(A-\mathcal{G P}) / K(A-\mathcal{P}))^{c}
$$

is fully faithful and identifies $D_{g p}^{b}\left(A^{\mathrm{op}}\right)^{\mathrm{op}} / D^{b}\left(A^{\mathrm{op}}\right)^{\mathrm{op}}$ with a subcategory of $(K(A-\mathcal{G P}) / K(A-\mathcal{P}))^{c}$ whose épaisse closure is all of $(K(A-\mathcal{G P}) / K(A-\mathcal{P}))^{c}$.

Proof. [G2, Theorem 2.6] implies (1). Now we prove (2). By Lemma 3.5, we know that $K(A-\mathcal{P})$ is a compactly generated triangulated category with $\mathcal{G}_{1}$ as a set of compact generators, and that there is an equivalence of triangulated categories $K(A-\mathcal{P})^{c} \cong$ $D^{b}\left(A^{\mathrm{op}}\right)^{\mathrm{op}}$. By Theorem 3.2 , we get that $K(A-\mathcal{G P})$ is a compactly generated triangulated category with $\mathcal{G}_{2}$ as a set of compact generators, and that there is an equivalence of triangulated categories $K(A-\mathcal{G P})^{c} \cong D_{g p}^{b}\left(A^{\mathrm{op}}\right)^{\mathrm{op}}$.

Let $\left\{G_{i}^{\bullet}\right\}_{i \in I}$ be any family of objects in $K(A-\mathcal{G P})$. Then $\operatorname{Hom}_{K(A-\mathcal{G P})}\left(i P, \coprod_{i \in I} G_{i}^{\bullet}\right)$ $=\operatorname{Hom}_{K(A-\mathcal{G P})}\left(P, \coprod_{i \in I} G_{i}^{\bullet}\right) \cong \coprod_{i \in I} \operatorname{Hom}_{K(A-\mathcal{G P})}\left(P, G_{i}^{\bullet}\right)=\coprod_{i \in I} \operatorname{Hom}_{K(A-\mathcal{G P})}\left(i P, G_{i}^{\bullet}\right)$ for each module $P \in A$-proj. So by [CFH, Proposition 2.6], the inclusion $i: K(A-\mathcal{P}) \hookrightarrow$ $K(A-\mathcal{G P})$ preserves compact objects.

Applying [N1, Theorem 2.1] to the homotopy category $K(A-\mathcal{G P})$ and $K(A-\mathcal{P})$, we get that $i$ carries $K(A-\mathcal{P})^{c}$ to $K(A-\mathcal{G P})^{c}, q$ carries $K(A-\mathcal{G P})^{c}$ to $(K(A-\mathcal{G P}) / K(A-\mathcal{P}))^{c}$, the natural functor $\widetilde{F}: K(A-\mathcal{G P})^{c} / K(A-\mathcal{P})^{c} \rightarrow(K(A-\mathcal{G P}) / K(A-\mathcal{P}))^{c}$ is fully faithful, and any object in $K(A-\mathcal{G P})^{c} / K(A-\mathcal{P})^{c}$ is a direct summand of some object in $(K(A-\mathcal{G P}) / K(A-\mathcal{P}))^{c}$. This completes the proof of $(2)$.

Recall that an additive category $\mathcal{C}$ is idempotent-complete if every idempotent morphism splits. Any additive category admits an idempotent completion $l: \mathcal{C} \rightarrow \mathcal{C}^{\natural}$. Moreover, if $\mathcal{C}$ is triangulated, then $\mathcal{C}^{\natural}$ inherits a unique structure of triangulated category such that $l$ is a triangle functor(see [BS1, Theorem 1.5]). 
Remark 3.7. Let $A$ be a virtually Gorenstein algebra of finite CM-type. Theorem 3.6 implies that the idempotent completion of $D_{g p}^{b}\left(A^{\mathrm{op}}\right)^{\mathrm{op}} / D^{b}\left(A^{\mathrm{op}}\right)^{\mathrm{op}}$ and $(K(A-\mathcal{G P})$ $/ K(A-\mathcal{P}))^{c}$ are triangluar equivalent, and also that $D^{b}(A)$ can be viewed as a triangulated subcategory of $D_{g p}^{b}(A)$. But in general, this doesn't hold.

In general, a Gorenstein flat module is not necessarily Gorenstein projective for a ring. Next, we will establish a pair of adjoint functors between the homotopy category of Gorenstein flat modules and the homotopy category of a nice ring.

Theorem 3.8. Let $R$ be a two-sided noetherian ring such that $R-\mathcal{G F}$ is closed under direct products. Then the inclusion $K(R-\mathcal{G F}) \rightarrow K(R-\mathrm{Mod})$ admits a right adjoint.

Proof. By the proof of [EEI, Theorem 4.3], we get that every complex $X^{\bullet}$ in $K(R$-Mod) admits a $K(R-\mathcal{G F})$-precover. Hence by [N5, Proposition 1.4] we get that the inclusion $K(R-\mathcal{G} \mathcal{F}) \rightarrow K(R$-Mod $)$ admits a right adjoint.

Now we show an interesting phenomenon. Let $M$ be any $R$-module, and consider the complex

$$
\cdots \rightarrow 0 \rightarrow M \rightarrow 0 \rightarrow \cdots .
$$

The existence of a right adjoint to the inclusion gives us a morphism $Z^{\bullet} \rightarrow M$, in the category $K(R$-Mod),

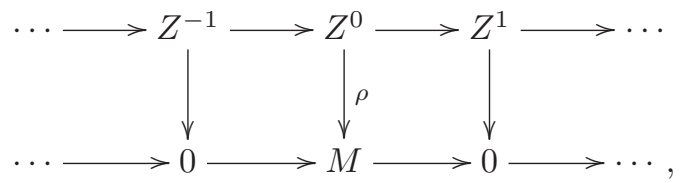

where the complex $Z^{\bullet}$ is a complex of Gorenstein flat $R$-modules. Furthermore, given any map $\varphi: F^{\bullet} \rightarrow M$, with $F$ a Gorenstein flat $R$-module, we have a factorization of $\varphi$ :

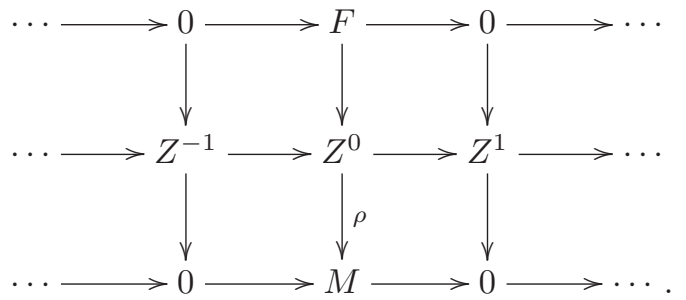

This shows the map $\rho: Z^{0} \rightarrow M$ is a Gorenstein flat precover for $M$.

Corollary 3.9. Let $R$ be a two-sided noetherian ring such that $R-\mathcal{G} \mathcal{F}$ is closed under direct products. Then the inclusion $i: K(R-\mathcal{F}) \rightarrow K(R-\mathcal{G} \mathcal{F})$ has a right adjoint.

Proof. By [N5, Theorem 3.2], we get that the inclusion $i_{1}: K(R-\mathcal{F}) \rightarrow K(R$-Mod $)$ has a right adjoint $j_{1}$. By Theorem 3.8, we get that the inclusion $i_{2}: K(R-\mathcal{G F}) \rightarrow$ $K(R$-Mod $)$ has a right adjoint $j_{2}$. Since we have a series of isomorphisms for any 


$$
\begin{aligned}
F^{\bullet} \in K(R-\mathcal{F}) \text { and } Z^{\bullet} & \in K(R-\mathcal{G} \mathcal{F}) \\
\operatorname{Hom}_{K(R-\mathcal{G F})}\left(i F^{\bullet}, Z^{\bullet}\right) & \cong \operatorname{Hom}_{K(R \text {-Mod })}\left(i_{2} i F^{\bullet}, i_{2} Z^{\bullet}\right)=\operatorname{Hom}_{K(R \text {-Mod })}\left(i_{1} F^{\bullet}, i_{2} Z^{\bullet}\right) \\
& \cong \operatorname{Hom}_{K(R-\mathcal{F})}\left(F^{\bullet}, j_{1} i_{2} Z^{\bullet}\right),
\end{aligned}
$$

it follows that $i: K(R-\mathcal{F}) \rightarrow K(R-\mathcal{G} \mathcal{F})$ admits a right adjoint $j_{1} i_{2}$.

\section{Gorenstein representation dimension}

In this section, we introduce the notion of Gorenstein representation dimension for an algebra of finite CM-type and establish the relations between it and the dimension of its bounded Gorenstein derived category.

Let $\mathcal{T}$ be a triangulated category, and let $M \in \mathcal{T}$. We set

$$
\begin{gathered}
\langle M\rangle=\langle M\rangle_{1}=\operatorname{add}\{M[i] \mid i \in \mathbb{Z}\} \\
\langle M\rangle_{n+1}=\operatorname{add}\left\{X \mid \exists M^{\prime} \rightarrow X \rightarrow M^{\prime \prime} \rightarrow M^{\prime}[1] \text { with } M^{\prime} \in\langle M\rangle, M^{\prime \prime} \in\langle M\rangle_{n}\right\} .
\end{gathered}
$$

Recall from $[\mathrm{Ro}]$ that the dimension of a triangulated category $\mathcal{T}$ is the number

$$
\operatorname{dim} \mathcal{T}=\inf \left\{n \in \mathbb{N} \mid \text { there exists a } M \in \mathcal{T} \text { with }\langle M\rangle_{n+1}=\mathcal{T}\right\} .
$$

For a subcategory $\mathcal{C} \subseteq \mathcal{T}$, the dimension is defined to be $\operatorname{dim}_{\mathcal{T}} \mathcal{C}=\inf \{n \mid \exists M \in \mathcal{T}$ such that $\left.\mathcal{C} \subseteq\langle M\rangle_{n+1}\right\}$.

Suppose $A$ is an artin algebra of finite CM-type. Let $G_{1}, \cdots, G_{n}$ be all the pairwise non-isomorphic indecomposable finitely generated Gorenstein projective $A$-modules. Set $G=\bigoplus_{1 \leqslant i \leqslant n} G_{i}$, and $\mathcal{G} p(A):=\operatorname{End}_{A}(G)^{\mathrm{op}}$. We first have the following:

Lemma 4.1. Let $A$ be a finite-dimensional $k$-algebra of finite $C M$-type over a field $k$ and $\mathcal{G} p \operatorname{dim} X=n$ for some $X \in A$-mod. Then $X \notin\langle G\rangle_{n}$.

Proof. The proper $A-\mathcal{G}$ proj-resolution

$$
\Omega_{G}^{n} X \longmapsto G_{n-1} \rightarrow G_{n-2} \rightarrow \cdots \rightarrow G_{1} \rightarrow G_{0} \rightarrow X
$$

gives rise to a sequence of maps

$$
X \rightarrow \Omega_{G} X[1] \rightarrow \cdots \rightarrow \Omega_{G}^{n-1} X[n-1] \rightarrow \Omega_{G}^{n} X[n]
$$

in $D_{g p}^{b}(A)$. They are all $\langle G\rangle$-ghosts, and their composition is non-zero. Hence the claim follows from the ghost lemma in the sense of Rouquier [Ro].

So we try to introduce the notion of Gorenstein representation dimension. Of particular interest to us are $\operatorname{dim} D_{g p}^{b}(A)$ and $\operatorname{dim}_{D_{g p}^{b}(A)}(A$-mod).

Definition 4.2. Let $A$ be an artin algebra of finite CM-type. The Gorenstein representation dimension of $A$ is defined as

$$
\begin{aligned}
\operatorname{Grepdim} A=\min \{M \text {-resol.dim}(A \text {-mod }) \mid & M \in A \text {-mod } \\
& \text { such that } G \oplus \nu(G) \in \operatorname{add} M\}+2 .
\end{aligned}
$$

An $A$-module $M$ realizing the minimum above is called the Gorenstein Auslander generator. 
Remark 4.3. Let $A$ be an $\operatorname{artin}$ algebra of finite CM-type. Then $\operatorname{repdim} A \leqslant \operatorname{Grepdim} A$ by the definition of representation dimension (see $[\mathrm{Au}]$ ). Also, if $A$ is CM-free (i.e., $A-\mathcal{G P}=A-\mathcal{P})$, then these two definitions coincide.

Lemma 4.4. Let $A$ be an artin algebra of finite CM-type. Then

Grepdim $A=\min \left\{\right.$ gl.dimEnd ${ }_{A}(M) \mid M \in A-\bmod$ such that $\left.G \oplus \nu(G) \in \operatorname{add} M\right\}$.

Proof. For any $M \in A$-mod with $G \oplus \nu(G) \in \operatorname{add} M$, we easily see that $M$ is a generator and a cogenerator. Hence the claim follows from [Au].

Now we will establish relations among above-mentioned dimensions for an algebra of finite CM-type.

Theorem 4.5. Let $A$ be a finite-dimensional k-algebra of finite CM-type. Let $M \in$ $A$-mod be a Gorenstein Auslander generator, and let $X \in A$-mod. Then for any integer $n \geqslant 0$, we have

$$
M \text {-resol.dim } X \leqslant n \Longrightarrow X \in\langle M\rangle_{n+1} \text {. }
$$

In particular,

$$
\operatorname{Grepdim} A \geqslant \operatorname{dim}_{D_{g p}^{b}(A)}(A-\bmod )+2 .
$$

Proof. Let $\varepsilon: 0 \rightarrow X \rightarrow Y \rightarrow Z \rightarrow 0$ be a short exact sequence in $A$-mod with $\mathrm{Hom}_{A}$ $(G, \varepsilon)$ exact for any A-module $G$ in $A$ - $\mathcal{G}$ proj. Then by the definition of $D_{g p}^{b}(A)$, we get that $X \rightarrow Y \rightarrow Z \rightarrow X[1]$ is a distinguished triangle in $D_{g p}^{b}(A)$. This implies that $M$-resol.dim $X \leqslant n \Longrightarrow X \in\langle M\rangle_{n+1}$. Thus Grepdim $A \geqslant \operatorname{dim}_{D_{g p}^{b}(A)}(A$-mod $)+2$.

Theorem 4.6. Let $A$ be a finite-dimensional k-algebra of finite CM-type. Then

$$
\operatorname{Grepdim} A \geqslant \operatorname{dim} D_{g p}^{b}(A) \text {. }
$$

Proof. Let $M$ be a Gorenstein Auslander generator. By induction on the length of the bounded complex of $A$-mod, we get that every bounded complex of $A$-mod is $A-\mathcal{G}$ proj-quasi-isomorphic to a bounded complex of add $M$, i.e., the canonical functor $K^{b}(\operatorname{add} M) \rightarrow D_{g p}^{b}(A)$ is essentially surjective. Notice that we have canonical equivalences $K^{b}(\operatorname{add} M) \cong K^{b}\left(\operatorname{End}_{A}(M)-\operatorname{proj}\right) \cong D^{b}\left(\operatorname{End}_{A}(M)\right)$ and that $\operatorname{dim} D^{b}\left(\operatorname{End}_{A}(M)\right)$ $\leqslant$ gl.dimEnd $A(M)$. We deduce that $\operatorname{Grepdim} A \geqslant \operatorname{dim} D_{g p}^{b}(A)$.

This completes the proof.

\section{Acknowledgments}

Some of this work was done when the author was visiting Professor Steffen König at the University of Stuttgart, Germany. The author would like to thank Steffen König for useful discussions and comments related to this work.

\section{References}

[Au] M. Auslander, Representation dimension of Artin algebras, Queen Mary College Math. Notes, London, 1971.

[ARS] M. Auslander, I. Reiten, and S. O. Smalø, Representation Theory of Artin Algebras, Cambridge Studies in Adv. Math. 36, Cambridge Univ. Press, Cambridge, 1995. 
[AM] L.L. Avramov and A. Martsinkovsky, Absolute, relative, and Tate cohomology of modules of finite Gorenstein dimension, Proc. London Math. Soc. 85 (2002), 393-440.

[BS1] P. Balmer and M. Schlichting, Idempotent completion of triangulated categories, J. Algebra 236 (2001), 819-834.

[BS2] S. Bazzoni and J. Stovicek, On the abelianization of derived categories and a negative solution to Rosicky's problem, Compos. Math. 149 (2013), $125-147$.

[Be] A. Beligiannis, On algebras of finite Cohen-Macaulay type, Adv. Math. 226 (2011), 1973-2019.

[BR] A. Beligiannis and I. Reiten, Homological and homotopical aspects of tosion theories, Mem. Amer. Math. Soc. 188, Amer. Math. Soc., 2007.

[BIKO] P.A. Bergh, S.B. Iyengar, H. Krause, and S. Oppermann, Dimensions of triangulated categories via Koszul objects, Math. Z. 265 (2010), 849-864.

[BH] W. Bruns and J. Herzog, Cohen-Macaulay Rings, Revised edition, Cambridge Studies in Adv. Math. 39, Cambridge Univ. Press, Cambridge, 1998.

[Buc] R.O. Buchweitz, Maximal Cohen-Macaulay modules and TateCohomology over Gorenstein rings, Unpublished manuscript, 1987.

[CN] C. Casacuberta and A. Neeman, Brown representability does not come for free, Math. Res. Lett. 16 (2009), 1-5.

[CFH] L.W. Christensen, A. Frankild, and H. Holm, On Gorenstein projective, injective and flat dimensions - a functorial description with applications, J. Algebra 302 (2006), 231-279.

[CPS] E. Cline, B. Parshall, and L. Scott, Algebraic stratification in representation categories, J. Algebra 117 (1988), 504-521.

[EE] E. Enochs and S. Estrada, Relative homological algebra in the category of quasi-coherent sheaves, Adv. Math. 194 (2005), 284-295.

[EEG-R] E. Enochs, S. Estrada, and J.R. García-Rozas, Gorenstein categories and Tate cohomology on projective schemes, Math. Nachr. 281 (2008), $525-540$.

[EEI] E. Enochs, S. Estrada, and A. Iacob, Gorenstein projective and flat complexes over noetherian rings, Math. Nachr. 285 (2012), 834-851.

[EJ] E.E. Enochs and O.M.G. Jenda, Relative Homological Algebra, De Gruyter Exp. Math. 30, Walter De Gruyter Co., 2000.

[EX] E.E. Enochs and J.Z. Xu, Gorenstein flat covers of modules over Gorenstein rings, J. Algebra 181 (1996), 288-313.

[G1] N. Gao, Stable t-structures and homotopy category of Gorensteinprojective modules, J. Algebra 324 (2010), 2503-2511.

[G2] N. Gao, A smashing subcategory of the homotopy category of Gorenstein projective modules, Appl. Categor. Struct. 23 (2015), 87-91.

[GZ1] P. Gabriel and M. Zisman, Calculus of Fractions and Homotopy Theory, Springer, New York, 1967. 
[GZ2] N. Gao and P. Zhang, Gorenstein derived categories, J. Algebra 323 (2010), 2041-2057.

[HJ1] H. Holm and P. Jørgensen, Compactly generated homotopy categories, Homology, Homotopy Appl. 9 (2007), 257-274.

[HJ2] H. Holm and P. Jørgensen, Rings without a Gorenstein analogue of the Govorov-Lazard theorem, Q. J. Math. 62 (2011), 977-988.

[J] P. Jørgensen, The homotopy category of complexes of projective modules, Adv. Math. 193 (2005), 223-232.

[K1] H. Krause, The stable derived category of a Noetherian scheme, Compos. Math. 141 (2005), 1128-1162.

[K2] H. Krause, Approximations and adjoints in homotopy categories, Math. Ann. 353 (2012), 765-781.

[KK] H. Krause and D. Kussin, Rouquier's theorem on representation dimension, in Trends in Representation Theory of Algebras and Related Topics, in Contemp. Math. 406, 95-103, Amer. Math. Soc., Providence, RI, 2006.

[LO] V.A. Lunts and D.O. Orlov, Uniqueness of enhancement for triangulated categories, J. Amer. Math. Soc. 23 (2010), 853-908.

[Mi] J. Miyachi, Localization of triangulated categories and derived categories, J. Algebra 141 (1991), 463-483.

[N1] A. Neeman, The connection between the K-theory localization theorem of Thomason, Trobaugh and Yao and the smashing subcategories of Bousfield and Ravenel, Ann. Scient. Éc. Norm. Sup. 25 (1992), 547-566.

[N2] A. Neeman, The Grothendieck duality theorem via Bousfield's techniques and Brown representability, J. Amer. Math. Soc. 8 (1996), 205-236.

[N3] A. Neeman, Triangulated Categories, Annals of Mathematics Syudies 148, Princeton Univ. Press, 2001.

[N4] A. Neeman, The homotopy category of flat modules, and Grothendieck duality, Invent. Math. 174 (2008), 255-308.

[N5] A. Neeman, Some adjoints in homotopy categories, Ann. Math. 171 (2010), 2143-2155.

[O] S. Oppermann, Lower bounds for Auslander's representation dimension, Duke Math. J. 148 (2009) 211-249.

[Ro] R. Rouquier, Dimension of triangulated categories, J. K-Theory 1 (2008), 193-256.

[S] S. Schwede, The stable homotopy category is rigid, Ann. Math. 166 (2007), 837-863.

[Ver] J.L. Verdier, Catégories dérivées, etat 0, in Lecture Notes in Math. 569, 262-311, Springer-Verlag, 1977.

Nan Gao nangao@shu.edu.cn

Department of Mathematics, Shanghai University, Shangda Road 99, Shanghai, 200444, P. R. China 